\title{
Estimates and Extremals for Zeta Function Determinants on Four-Manifolds ${ }^{\star}$
}

\author{
Thomas P. Branson ${ }^{1}$, Sun-Yung A. Chang ${ }^{2}$ and Paul C. Yang ${ }^{3}$ \\ 1 Department of Mathematics, The University of Iowa, Iowa City, IA 52242, USA, Sonder- \\ forschungsbereich 170: "Geometrie und Analysis", Bunsenstrasse 3-5, W-3400 Göttingen, FRG, \\ and Matematisk Institut, Odense Universitet, DK-5230 Odense M, Denmark; E-mail address: \\ branson@math.uiowa.edu \\ 2 Department of Mathematics, UCLA, 405 Hilgard Avenue, Los Angeles, CA 90024, USA; \\ E-mail address: chang@math.ucla.edu \\ ${ }^{3}$ Department of Mathematics, University of Southern California, Los Angeles, CA 90089-1113, \\ USA; E-mail address: pyang\%mtha.usc.edu@usc.edu
}

Received May 6, 1991; in revised form April 7, 1992

\begin{abstract}
Let $A$ be a positive integral power of a natural, conformally covariant differential operator on tensor-spinors in a Riemannian manifold. Suppose that $A$ is formally self-adjoint and has positive definite leading symbol. For example, $A$ could be the conformal Laplacian (Yamabe operator) $L$, or the square of the Dirac operator $\not$. Within the conformal class $\left\{g=e^{2 w} g_{0} \mid w \in C^{\infty}(M)\right\}$ of an Einstein, locally symmetric "background" metric $g_{0}$ on a compact four-manifold $M$, we use an exponential Sobolev inequality of Adams to show that bounds on the functional determinant of $A$ and the volume of $g$ imply bounds on the $W^{2,2}$ norm of the conformal factor $w$, provided that a certain conformally invariant geometric constant $k=k\left(M, g_{0} A\right)$ is strictly less than $32 \pi^{2}$. We show for the operators $L$ and $\not \nabla^{2}$ that indeed $k<32 \pi^{2}$ except when $\left(M, g_{0}\right)$ is the standard sphere or a hyperbolic space form. On the sphere, a centering argument allows us to obtain a bound of the same type, despite the fact that $k$ is exactly equal to $32 \pi^{2}$ in this case. Finally, we use an inequality of Beckner to show that in the conformal class of the standard four-sphere, the determinant of $L$ or of $\not^{2}$ is extremized exactly at the standard metric and its images under the conformal transformation group $O(5,1)$.
\end{abstract}

\section{Introduction and Statement of Results}

On a compact Riemannian manifold $(M, g)$, there are many natural, or geometric elliptic operators associated with the metric; for example the Laplacian $\Delta=-g^{-1 / 2} \partial_{i}\left(g^{i j} g^{1 / 2} \partial_{j}\right)$ on functions. When we wish to emphasize the underlying conformal structure, it is natural to consider operators which transform in a simple manner under conformal change of metric. A conformally covariant operator is a geometric differential operator $A$ which undergoes the following transformation

\footnotetext{
* The first-named author acknowledges support from the University of Iowa Center for Advanced Studies, Odense Universitet, and Sonderforschungsbereich 170: "Geometrie und Analysis" in Göttingen. Research of the second- and third-named authors was partially supported by the NSF
} 
when the metric is changed conformally by $\bar{g}=e^{2 w} g, w \in C^{\infty}(M)$ :

$$
\bar{A}=e^{-b w} A e^{a w}
$$

for some constants $a$ and $b$. Here the $e^{a w}$ on the right is to be viewed as a multiplication operator. In dimension two it is well known that the Laplacian $\Delta$ on functions satisfies (1.1) with $a=0$ and $b=2$. In general dimension $n>1$ the conformal Laplacian $L=\Delta+(n-2) \tau / 4(n-1)$, where $\tau$ is the scalar curvature of the metric, is the simplest operator enjoying a conformal covariance property: under the change of metric $g_{w}=e^{2 w} g_{0}$ we have (1.1) with $a=(n-2) / 2$ and $b=(n+2) / 2$. A closely related operator is the Dirac operator on sections of the spinor bundle $\Sigma$. When $M$ carries a spin structure, the Dirac operator $\phi=\gamma^{i} \cdot \nabla_{i}$, where $\omega^{1}, \ldots, \omega^{n}$ form a local orthonormal frame for the cotangent bundle $T^{*} M ; \gamma$ is the fundamental section of $T M \otimes \Sigma \otimes \Sigma^{*} ; \gamma^{i}=\gamma\left(\omega^{i}\right)$ (a local section of $\Sigma \otimes \Sigma^{*} \cong$ End $\Sigma$ ); and $\nabla$ is the Riemannian connection lifted to $\Sigma$. Relative to the explicit construction of $\Sigma$ via the Clifford algebra of the tangent bundle $T M$, the $\gamma^{i}$ act as elements of the Clifford algebra on the Clifford module of spinors. The $\gamma^{i}$ satisfy the Clifford relations $\gamma^{i} \gamma^{j}+\gamma^{j} \gamma^{i}=-2 g^{i j} I$, where $I$ is the identity endomorphism, as well as the condition $\nabla \gamma=0$. The Dirac operator enjoys the conformal covariance property (1.1) with $a=(n-1) / 2, b=(n+1) / 2$ :

$$
\bar{g}=e^{2 w} g, \quad \bar{\gamma}=e^{-w} \gamma \Rightarrow \bar{\phi}=e^{-\frac{n+1 w}{2} \not} \not e^{-\frac{n-1}{2} w} .
$$

These elliptic operators have discrete spectrum $\left\{\lambda_{k}\right\}$. It is by now a well-known problem to control the metric using the spectral data of its Laplacian, or some other natural differential operator. McKean and Singer [MS] showed how to relate this spectral data to local invariants of the metric through consideration of the trace of the heat kernel. More recently, the determinant of the Laplacian first introduced by Ray and Singer [RS] in their study of the Reidmeister torsion has played a role in this problem. In dimension two, Osgood, Philips, and Sarnak have made essential use of the zeta function determinant and a sharp inequality of Moser to show that the Laplace spectrum determines the metric up to a compact set in the $C^{\infty}$ topology. Prior to the compactness result, Onofri and also Osgood-Philips-Sarnak showed the following:

Theorem [On, OPS1]. On a compact surface with background metric $g_{0}$, among all conformal metrics $g_{w}=e^{2 w} g_{0}$ of fixed area, the metric of constant curvature minimizes the quantity $-\log \operatorname{det}(\Delta)$.

In connection with the isospectral problem, there is the following preliminary bound:

Theorem [OPS2]. On a compact surface with a background metric $g_{0}$, consider a conformal metric $g_{w}=e^{2 w} g_{0}$. There exists a constant $C=C\left(\zeta_{\Delta}^{\prime}(0)\right.$, area $\left.\left(g_{w}\right)\right)$ such that

$$
\int|d w|^{2} \leqq C
$$

In this article we explore this phenomenon in dimension four and consider the natural operators $L$ and $\phi^{2}$. Our treatment is actually broad enough to encompass natural operators of the form $A=B^{p}$, where $B$ is conformally covariant, $A$ is formally self-adjoint with positive definite leading symbol, and $p$ is a positive integer. In general it is not possible to relate the highly global log-determinant invariant $\zeta_{A}^{\prime}(0)$ to local invariants of the operator $A$. However, in case the operator 
$A$ is a positive power of a conformal covariant as immediately above, it is possible to express the difference of log-determinants in this way, as long as we stay within a conformal class. Polyakov has shown that for compact surface with conformal metrics $g_{w}=e^{2 w} g_{0}$, we have

$$
\zeta_{\Delta_{w}}^{\prime}(0)-\zeta_{\Delta_{0}}^{\prime}(0)=\frac{1}{3} \int\left\{|d w|^{2}+\tau_{0} w\right\}
$$

where $\tau_{0}$ is the scalar curvature (twice the Gauss curvature) of $g_{0}$. This formula has a natural generalization to dimension four given by Branson and Ørsted [BØ3] for compact manifolds with locally symmetric, Einstein background metrics; see Proposition 2.5. This is a broader class of spaces than just the constant-curvature space forms; there are seven possibilities for the universal cover of such a space (see Sect. 3).

Our first aim is to use the log-determinant to obtain a bound for the conformal factor. For this we require an analogue of the Moser-Trudinger inequality for higher-order derivatives, a sharp version of which was given by Adams [Ad, Theorem 1]. In dimension four, this takes the following form: Let $\Omega$ be a bounded domain in $\mathbb{R}^{4}, u \in C_{0}^{2}(\Omega), \int|\Delta u|^{2} \leqq 1$; there exists a constant $c_{0}$ such that

$$
\int_{\Omega} e^{32 \pi^{2} u^{2}} \leqq c_{0}|\Omega|
$$

This inequality can be adapted to compact 4-manifolds. (For general compact manifolds, the corresponding inequality in some special cases has been worked out by Fontana $[\mathrm{F}]$.) Using the resulting inequality, we prove:

Theorem 1.1. Suppose $\left(M, g_{0}\right)$ is a 4-dimensional compact locally symmetric Einstein manifold which is neither the standard 4-sphere nor a hyperbolic sapce form. Consider a conformal metric $g_{w}=e^{2 w} g_{0}$. There exists constants $C=C_{L}\left(\operatorname{vol}(g), \zeta_{L}^{\prime}(0)\right)$ and $C=C_{\nabla^{2}}\left(\operatorname{vol}(g), \zeta_{\not^{2}}^{\prime}(0)\right)$ such that

$$
\|w\|_{2,2} \leqq C .
$$

In case $\left(M, g_{0}\right)$ is the standard 4-sphere, there is a suitable conformal transformation $\phi$ in the conformal diffeomorphism group $\mathscr{C}\left(\mathbb{S}^{4}, g_{0}\right)$ so that (1.3) holds for the transformed conformal factor $w_{\phi}$ given by $e^{2 w_{\phi}} g_{0}=\phi^{*}\left(e^{2 w} g_{0}\right)$.

As a consequence we can study the extremals of the zeta function determinant of the conformal Laplacian and the square of the Dirac operator for conformal metrics. It follows that a minimizing sequence converges to a solution of a fourthorder equation. In case of the 4-sphere we can show that the minimum is in fact attained at the standard metric:

Theorem 1.2. On the standard 4-sphere $\left(\mathbb{S}^{4}, g_{0}\right)$, the standard metric $g_{0}$ minimizes $\log \operatorname{det}(L)$, as well as - $\log \operatorname{det}\left(\not^{2}\right)$, among all conformal metrics of fixed volume.

For the proof, a sharp inequality discovered by Beckner is essential: Expanding $f \in C^{\infty}\left(\mathbb{S}^{n}\right)$ in spherical harmonics $f=\sum_{k=0}^{\infty} Y_{k}$, we have

$$
\log \underset{\mathbb{S}^{n}}{f} e^{f-\bar{f}} \leqq \frac{1}{2 n} \sum_{k=1}^{\infty} \frac{\Gamma(n+k)}{\Gamma(n) \Gamma(k)} \underset{\mathbb{S}^{n}}{f}\left|Y_{k}\right|^{2},
$$

where $f_{\mathbb{S}^{n}}$ is the normalized integral $\left(f_{\mathbb{S}^{n}} 1=1\right)$. Equality holds if and only if $e^{2 f / n} g_{0}=\phi^{*} g_{0}$ for some conformal transformation $\phi$ of the standard sphere. 
We give here an outline of the remainder of the paper. In Sect. 2 we give the adaptation of the Adams inequality to compact manifolds and prove a general bound for $\|w\|_{2,2}$ under the assumption that a certain constant $k=k\left(M, g_{0}, A\right)$ associated to the operator $A$ and the manifold $\left(M, g_{0}\right)$ is strictly less than $32 \pi^{2}$.

In Sect. 3 we verify that for the operators $L$ and $\not^{2}$, this constant is smaller than $32 \pi^{2}$ for all compact locally symmetric Einstein 4-manifolds except the hyperbolic space forms and the 4-sphere. In Sect. 4 we employ a device introduced by Aubin to show that even in the case $M=\mathbb{S}^{4}$, where $k=32 \pi^{2}$, such a bound is still possible after suitable conformal transformation. Finally in Sect. 5 we give the proof of Theorem 1.2 using the inequality of Beckner.

We would like to thank Bill Beckner and Bent Ørsted for enlightening conversations, and Peter Sarnak for his interest in this work.

\section{The Adams Inequality, the Functional Determinant, and the $W^{2,2}$ Bound}

In this section we shall establish a uniform $W^{2,2}$ bound on the conformal factors $w\left(g=e^{2 w} g_{0}\right)$, valid for some compact 4-manifolds without boundary, under the assumption that the $\log$ determinant $F[w]=\log \left(\operatorname{det} L(w) / \operatorname{det} L_{0}(w)\right)$ is fixed. Our argument will show that the conformal Laplacian $L$ can be replaced by an operator from a general class which includes the square $\not^{2}$ of the Dirac operator. A basic analytic fact which we shall use is the following generalization of Moser's inequality due to D. Adams.

Proposition 2.1. [Ad, Theorem 1] Let $m<n$ be positive integers, and let $\Omega$ be a bounded domain in $\mathbb{R}^{n}$. There are constants $c_{0}=c_{0}(m, n)$ and $\beta_{0}=\beta_{0}(m, n)$ such that for all $u \in C^{m}\left(\mathbb{R}^{n}\right)$ with support contained in $\Omega$ and $\left\|\nabla^{m} u\right\|_{p} \leqq 1, p=n / m$,

$$
\int_{\Omega} \exp \left(\beta|u(x)|^{p^{\prime}}\right) d x \leqq c_{0}|\Omega|
$$

for all $\beta \leqq \beta_{0}$, where $p^{\prime}=p /(p-1)$ and $|\Omega|$ is the measure of $\Omega$. Furthermore, if $\beta>\beta_{0}$, then there exists a smooth function $u$ supported in $\Omega$ with $\left\|\nabla^{m} u\right\|_{p} \leqq 1$ for which the integral in (2.1) can be made as large as we please.

[Ad, Theorem 1] gives an exact formula for $\beta_{0}$; in the special case where we shall apply the Adams inequality, $n=4, m=p=p^{\prime}=2$, and $\beta_{0}=\beta_{0}(2,4)=32 \pi^{2}$. For this special case, we shall adapt the inequality and its proof to functions defined on a compact 4-dimensional manifold without boundary. In this setting, the inequality takes the following form.

Proposition 2.2. Let $(M, g)$ be a 4-dimensional compact Riemannian manifold without boundary. There is a constant $c_{0}$ such that for all $u \in C^{2}(M)$ with $\|\Delta u\|_{2} \leqq 1$, we have

$$
\int_{M} \exp \left(32 \pi^{2}|u(x)-\bar{u}|^{2}\right) d v(x) \leqq c_{0} V(M),
$$

where $d v$ is the Riemannian measure determined by $g, \bar{u}=V(M)^{-1} \int_{M} u d v$, and $V(M)=\int_{M} d v$ is the volume of $M$. Furthermore the exponent $32 \pi^{2}$ is the best possible in the sense that if $\beta>32 \pi^{2}$, there exists a smooth function $u$ on $M$ with $\|\Delta u\|_{2} \leqq 1$ and $\int_{M} \exp \left(\beta|u(x)-\bar{u}|^{2}\right) d v(x)$ arbitrarily large.

As mentioned before, the proof of Proposition 2.2 below will be a modified version of Adams' proof of Proposition 2.1. For this reason, we shall only sketch 
the proof,' with special attention to the necessary modifications. Note that without the assumption that $\|\Delta u\|_{2} \leqq 1$, the inequality of Proposition 2.2 reads

$$
\int_{M} \exp \left(\frac{32 \pi^{2}|u(x)-\bar{u}|^{2}}{\|\Delta u\|_{2}^{2}}\right) d v(x) \leqq c_{0} V(M),
$$

as long as $u$ is not constant.

The following key lemma of Aubin replaces the fractional integral representation of compactly supported functions on $\mathbb{R}^{n}$ with a Green's function representation formula valid for functions on compact manifolds.

Lemma 2.3. [Au1, Theorem 4.13] Let $(M, g)$ be a compact, $n$-dimensional Riemannian manifold. There exists a Green's function $G(x, y)$ of the Laplacian $\triangle$ with the following properties:

(a) For all $C^{2}$ functions $u$ on $M$,

$$
u(x)-\bar{u}=\int_{M}(\Delta u(y)) G(x, y) d v(y) ;
$$

(b) $G(x, y)=G(y, x)$ is $C^{\infty}$ on the complement of the diagonal in $M \times M$;

(c) If $2 k>n>2$,

$$
G(x, y)=H(x, y)+\sum_{i=1}^{k} \int_{M} \Gamma_{i}(x, z) H(z, y) d v(y)+F(x, y),
$$

where $H(x, y)=\left((n-2) \omega_{n-1}\right)^{-1} r^{2-n} f(r)$ for $n>2 ; r=d(x, y)$ is the distance from $x$ to $y ; \omega_{n-1}=2 \pi^{n / 2} / \Gamma(n / 2)$ is the volume of the unit sphere in $\mathbb{R}^{n} ; f(r)$ is a positive decreasing function; $f(r)=1$ in a neighborhood of $r=0$; and $f(r)=0$ for $r \geqq \delta$, $\delta=\delta(M, g)$ being the imjectivity radius of $(M, g) ; \Gamma(x, y):=\Gamma_{1}(x, y)=-\Delta_{y} H(x, y)$;

$$
\Gamma_{i+1}(x, y)=\int_{M} \Gamma_{i}(x, z) \Gamma(z, y) d v(y), \quad i=1, \ldots, k-1
$$

and $F(x, y)$ is continuous on $M \times M$.

We remark that in the case of a Riemannian 4-manifold $(M, g)$, in the expression for the Green's function, we have a uniform estimate $|\Gamma(x, y)| \leqq \alpha r^{-2}$, valid when $r$ is less than the uniform injectivity radius $\delta$ [Au1, 4.10]. Here the constant $\alpha=\alpha(M, g)$ depends on uniform bounds for the sectional curvature of $g$ [Au1, 4.9 and Theorem 1.53]; like the uniform injectivity radius, such uniform bounds always exist for compact $M$. Thus if $Z_{i}(x, y):=\int_{M} \Gamma_{i}(x, z) H(z, y) d v(y)$,

$$
\left|Z_{1}(x, y)\right| \leqq \operatorname{const}(1+|\log d(x, y)|),
$$

and $Z_{2}(x, y)$ is continuous on $M \times M$. This implies that in the 4-dimensional case, we have

$$
G(x, y)=H(x, y)+Z(x, y)+R(x, y),
$$

where $|H(x, y)| \leqq\left(4 \pi^{2}\right)^{-1} r^{-2},|Z(x, y)| \leqq \operatorname{const}(1+|\log d(x, y)|)$, and $R(x, y)$ is continuous on $M \times M$.

Another basic lemma used in the proof of Proposition 2.2 is the following result of O'Neil. Suppose $f$ is a locally integrable function on $\mathbb{R}^{n}$, and denote 
by $f^{*}$ the symmetric decreasing rearrangement of $f$; that is, for each $s \geqq 0$, define $\lambda(s)=\left|\left\{x \in \mathbb{R}^{n}|| f(x) \mid>s\right\}\right|$, and define $f^{*}$ on $\mathbb{R}$ by $f^{*}(t)=\inf \{s>0 \mid \lambda(s) \leqq t\}$. Let $f^{* *}(t)=t^{-1} \int_{0}^{t} f^{*}(s) d s$.

Lemma 2.4. [O'N, Lemma 1.5] Let $h=T(f, g)=f * g$. Then

$$
h^{* *}(t) \leqq t f^{* *}(t) g^{* *}(t)+\int_{t}^{\infty} f^{*}(s) g^{*}(s) d s .
$$

To apply Lemma 2.4 in our setting, we just need to note that the proof of [O'N, Lemma 1.5] goes through if we replace the Euclidean convolution $f * g$ by

$$
h(x)=\int_{M} f(y) g(x, y) d v(y), \quad x \in M
$$

for a one-point function $f$ and a two-point function $g(x, y)=g(y, x)$ which is a function of $d(x, y)$. Here $f^{*}$ is defined by $f^{*}(t)=\inf \{s>0 \mid \lambda(s) \leqq t\}$, where $\lambda(s)=|\{x \in M|| f(x) \mid>s\}|$.

Proof of Proposition 2.2. Given $u \in C^{2}(M)$, apply (2.3) and (2.4) to write

$$
u(x)-\bar{u}=h(x)+z(x)+r(x),
$$

where $h(x)=\int_{M}(\Delta u)(y) H(x, y) d v(y), z(x)=\int_{M}(\Delta u)(y) Z(x, y) d v(y)$, and $\quad r(x)=$ $\int_{M}(\Delta u)(y) R(x, y) d v(y)$. Applying Hölder's inequality, we have

$$
|z(x)| \leqq\|\Delta u\|_{2}\left(\int_{M}|Z(x, y)|^{2} d v(y)\right)^{1 / 2} \leqq \alpha(M, g)
$$

for all $x \in M$. Hence $\|z\|_{\infty} \leqq \alpha(M, g)$. One can also apply a pointwise estimate to $r(x)$ to obtain $\|r\|_{\infty} \leqq \alpha(M, g)$. Thus

$$
\begin{aligned}
|u(x)-\bar{u}| & \leqq \int_{M}|(\Delta u)(y)||H(x, y)| d v(y)+\alpha(M, g) \\
& \leqq \frac{1}{4 \pi^{2}} \int_{M}|(\Delta u)(y)| d(x, y)^{-2} d v(y)+\alpha(M, g) .
\end{aligned}
$$

We now apply our adaptation of Lemma 2.4 with $f=|\Delta u|$ and $g(x, y)=d(x, y)^{-2} / 4 \pi^{2}$ to obtain

$$
\begin{aligned}
|u-\bar{u}|^{* *}(t) & \leqq \alpha(M, g)+h^{* *}(t) \\
& \leqq \alpha(M, g)+t f^{* *} g^{* *}(t)+\int_{t}^{\infty} f^{*}(s) g^{*}(s) d s,
\end{aligned}
$$

where again $h(x)=\int_{M} f(y) g(x, y) d v(y)$. From this point on, the argument of [Ad, Sect. 2] carries through to establish that

$$
\int_{M} \exp \left(32 \pi^{2}|u(x)-\bar{u}|^{2}\right) d v(x) \leqq \text { const } \cdot V(M) .
$$

We now wish to apply Proposition 2.2, in connection with a formula for the functional determinant in four dimensions due to Branson and Ørsted [BØ3], to establish the main result of this section. We digress briefly to sketch some background on functional determinants.

Let $A$ be a geometric partial differential operator with positive definite leading symbol and positive order. Then $A$ is automatically elliptic and of even order $2 l$. If 
$A$ is formally self-adjoint, it will have pure real eigenvalue spectrum $\left\{\lambda_{j} \mid j \in \mathbb{N}\right\}$ bounded below ( $\mathbb{N}$ denoting the natural numbers); in fact $\lambda_{j} \sim$ const $\cdot j^{2 l / n}$ as $j \uparrow \infty$. $A$ may have a finite number of nonpositive eigenvalues, but if $A$ is a positive integral power of a conformally covariant differential operator, the number of these (counted with multiplicity), as well as the multiplicity of 0 as an eigenvalue, are conformal invariants. The zeta function $\zeta_{A}(s)$ is defined for large $\operatorname{Re} s$ by

$$
\zeta_{A}(s)=\sum_{\lambda_{j} \neq 0}\left|\lambda_{j}\right|^{-s},
$$

and analytic continuation gives a meromorphic extension with isolated simple poles. $\zeta_{A}(s)$ is regular at the nonpositive integers. The zeta function determinant of $A$ is defined by

$$
\log |\operatorname{det} A|=-\zeta_{A}^{\prime}(0), \quad \operatorname{sign} \operatorname{det} A=(-1)^{\#\left\{\lambda_{j}<0\right\}} .
$$

For the analytic facts immediately above, and for more details on the asymptotics of the heat operator and the zeta function, we refer to [S, G].

There are other conventions in use for the handling of the zero and negative eigenvalues of $A$ in the definition of the determinant; it is just necessary to keep track of the effect of one's chosen convention on formulas like that of [BØ3]. In our situation, the sign of the determinant will be irrelevant, since we always deal with quotients of determinants of conformally related metrics; by the conformal invariance of $\#\left\{\lambda_{j}<0\right\}$, such quotients are always positive. For the most part, we shall need to know that 0 does not occur as an eigenvalue. For the positively curved backgrounds (with universal covers $\mathbb{S}^{4}, \mathbb{C P}^{2}$, and $\mathbb{S}^{2} \times \mathbb{S}^{2}$ ), we show in Sect. 3 that this is the case if our operator $A$ is $L$ or $\not^{2}$. If the background is flat (universal cover $\mathbb{R}^{4}$ ), zero modes cannot be avoided, but their effect on our formulas can be written down explicitly, so there is no problem here either. For the negatively curved backgrounds (with universal covers $\mathrm{H}^{4}, \mathbb{C H}^{2}$, and $\mathrm{H}^{2} \times \mathrm{H}^{2}$ ), we add $\mathcal{N}(A)=0$ as a side condition. In the derivation and treatment of the Polyakov formula (1.2) in dimension 2, the corresponding question was simpler: the Laplacian always had one zero mode, the constant functions.

Note that the determinant need not be invariant under uniform dilation of the metric:

$$
\bar{g}=c^{2} g, 0<c \in \mathbb{R} \Rightarrow \operatorname{det} \bar{A}=c^{-2 l \zeta_{A}(0)} \operatorname{det} A .
$$

In this sense the determinant comes in units of distance $-2 l \zeta_{A}(0)$. But this says exactly that the functional

$$
p_{A}(g)=\left(\frac{\operatorname{vol} g}{v_{0}}\right)^{2 l \zeta_{A}(0) / n} \operatorname{det} A,
$$

where $v_{0}$ is a positive constant, is scale-invariant. (Note that $\zeta_{A}(0)$ is also scaleinvariant.) As an alternative to looking at the functional $p_{A}$, we could freeze out scale changes by demanding that all our manifolds have volume $v_{0}$.

We can now state the four-dimensional Polyakov formula of Branson and Ørsted:

Proposition 2.5. [ВØ3, Proposition 2.1] Let $\left(M, g_{0}\right)$ be a compact, locally symmetric, Einstein 4-manifold without boundary, and let $w \in C^{\infty}(M)$. Suppose that $A$ is a formally self-adjoint positive integral power of a conformally covariant differential operator, that $A$ is orientation insensitive, and that $A$ has positive definite leading 
symbol of order $2 l \in 2 \mathbb{Z}^{+}$. If spin structure is required to define the bundle upon whose sections $A$ acts, assume that $\left(M, g_{0}\right)$ has such structure. Suppose that $A_{0}$ (that is, $A$ evaluated in $\left.\left(M, g_{0}\right)\right)$ has no zero spectrum. Then the functional determinant of $A_{1}$, that is, of $A$ evaluated in $\left(M, g_{1}=e^{2 w} g_{0}\right)$ is related to that of $A_{0}$ by sgn $\operatorname{det} A_{1}=\operatorname{sgn} \operatorname{det} A_{0}$, and, under the normalization $V(M, g)=\int_{M} e^{4 w}=v_{0}$,

$$
\begin{aligned}
\left(-\log \left|\operatorname{det} A_{1}\right|+\log \left|\operatorname{det} A_{0}\right|\right) / 2 l= & {\left[4 \pi^{2} \beta_{2} \chi(M)+\left(\beta_{1}-\frac{1}{8} \beta_{2}\right) v_{0} c^{2}\right] \frac{\int w}{v_{0}} } \\
& +\frac{1}{4} \beta_{2} \int(\Delta w)^{2}+\left(\frac{1}{4} \beta_{2}-\beta_{3}\right) J_{0} \int|d w|^{2} \\
& +\frac{1}{2} \beta_{3} \int\left(\frac{\Delta e^{w}}{e^{w}}\right)^{2}
\end{aligned}
$$

where $6 J_{0}$ and $c^{2}$ denote, respectively, the constant scalar curvature and constant norm-squared of the Weyl conformal curvature of $g_{0}$, all integrals are taken in the Riemannian measure determined by $g_{0}$, and $\beta_{1}, \beta_{2}$, and $\beta_{3}$ are constants which depend only on the operator $A$ (and not on $\left(M, g_{0}\right)$ ).

Remark 2.6. The constants $\beta_{1}, \beta_{2}, \beta_{3}$ originate in the asymptotics of the heat operator based on $A$ : suppose that $A$ satisfies all the conditions imposed above, except possibly the conformal condition. If $f \in C^{\infty}(M)$, there is an asymptotic expansion

$$
\operatorname{Tr}_{L^{2}} f \exp (-t A) \sim \sum_{i=0}^{\infty} t^{(2 i-n) / 2 l} \int f U_{i}[A], \quad t \downarrow 0,
$$

where the $U_{i}[A]$ are universal local scalar invariants. By Weyl's invariant theory, $U_{2}[A]$, and in fact every local scalar $O(4)$-invariant $S$ of homogeneity 4 (in the sense that uniform dilation $\tilde{g}=A^{2} g, 0<A \in \mathbb{R}$ of the metric produces the scaling $\left.\tilde{S}=A^{-4} S\right)$, is a linear combination of $|C|^{2},|B|^{2}, J^{2}$, and $\Delta J$, where $6 J$ is the scalar curvature $\tau, B$ is the Einstein (trace-free Ricci) tensor $\frac{1}{2}(\rho-\tau g / 4)(\rho=$ Ricci), $C$ is the Weyl conformal curvature tensor, $|B|^{2}=B^{i j} B_{i j}$, and similarly for $|C|^{2} . J, B$, and $C$ are (normalized versions of) the projections of the Riemann tensor $R$ onto the $O(4)$-irreducible summands of the vector bundle of algebraic curvature tensors. The orientation-insensitivity assumption is necessary to this statement, as $C$ breaks up into self- and anti-self-dual parts $C_{ \pm}$under the action of the structure group $S O(4)$ of oriented Riemannian geometry; this adds a new invariant, $\left|C_{+}\right|^{2}-\left|C_{-}\right|^{2}$, to our basis of the space of local scalar invariants of homogeneity 4 . A metric is conformally flat if $C=0$; Einstein if $B=0$, and locally symmetric if $\nabla R=0$ (or equivalently, if $\nabla J, \nabla B$, and $\nabla C$ all vanish). We shall use $V=B+J g / 4$ to simplify the appearance of some expressions. By [BØ2, Sect. 5], the assumption that $A$ is a positive integral power of a conformal invariant imposes one linear condition on the coefficients $a_{j}$ in the expression

$$
U_{2}[A]=a_{1}|C|^{2}+a_{2}|B|^{2}+a_{3} J^{2}+a_{4} \Delta J .
$$

One way of stating this condition which is especially well adapted to conformal change of metric is

$$
U_{2}[A]=\beta_{1}|C|^{2}+\beta_{2} Q+\beta_{3} \Delta J,
$$


where

$$
Q=-|V|^{2}+J^{2}+\frac{1}{2} \Delta J
$$

(See [BØ3, Sect. 2] for an account of the motivation behind this.) These are the constants $\beta_{j}$ in the statement of Proposition 2.5 above.

Remark 2.7. The simplest operators with the properties called for in the Proposition are the Yamabe operator, or conformal Laplacian $L$, and the square $\not^{2}$ of the Dirac operator $\phi$. These operators and their conformal properties are just as easy to describe in general dimension $n \geqq 2$ as they are in dimension four: $L=\Delta+(n-2) \tau / 4(n-1)$ acts on ordinary functions and enjoys the conformal covariance property

$$
\tilde{g}=e^{2 w} g, \quad w \in C^{\infty}(M) \Rightarrow \tilde{L}=e^{-(n+2) w / 2} L e^{(n-2) w / 2}
$$

on any Riemannian $n$-manifold $(M, g)$. (The $e^{(n-2) w / 2}$ on the right is to be understood as a multiplication operator.) $P$ acts on sections of the spinor bundle $\Sigma$ over a Riemannian spin manifold $(M, g, \gamma), \gamma$ being the fundamental section of $\Sigma \otimes \Sigma^{*} \otimes T M$, and enjoys the conformal covariance property

$$
\tilde{g}=e^{2 w} g, \quad \tilde{\gamma}=e^{-\omega} g, \quad w \in C^{\infty}(M) \Rightarrow \tilde{\nabla}=e^{-(n+1) w / 2} \not e^{(n-1) w / 2} .
$$

(The scaling law for $\gamma$ follows from that for $g$ together with the Clifford relations.) In [BØ3], the constants $\beta_{j}[L]$ and $\beta_{j}\left[\not^{2}\right]$ were computed from general formulas for $U_{2}[A]$; the result is

$$
\begin{aligned}
\left(4 \pi^{2}\right) \cdot 180\left(\beta_{1}, \beta_{2}, \beta_{3}\right)[L] & =(1,-4,-4), \\
\left(4 \pi^{2}\right) \cdot 360\left(\beta_{1}, \beta_{2}, \beta_{3}\right)\left[\not^{2}\right] & =(-7,88,28) .
\end{aligned}
$$

Remark 2.8. The analysis below is somewhat sensitive to the constants $\beta_{j}[A]$; specifically, it requires that $\beta_{2} \beta_{3}>0$ (recall that we have this for $A=L$ and $\left.A=\not \not^{2}\right)$. The value of $\beta_{1} / \beta_{2}$ also matters when $\left(M, g_{0}\right)$ is not conformally flat. With notation and assumptions as above, let

$$
k\left(M, g_{0}, A\right)=\left(4 \frac{\beta_{1}[A]}{\beta_{2}[A]}-\frac{1}{2}\right) v_{0} c^{2}+16 \pi^{2} \chi(M) .
$$

If $g_{0}$ is conformally flat, $k\left(M, g_{0}, A\right)=k(M)=16 \pi^{2} \chi(M)$ is independent of $g_{0}$ and $A$; in the conformally curved case,

$$
k\left(M, g_{0}, L\right)=-\frac{3}{2} v_{0} c^{2}+16 \pi^{2} \chi(M), \quad k\left(M, g_{0}, \not^{2}\right)=-\frac{9}{11} v_{0} c^{2}+16 \pi^{2} \chi(M) .
$$

We can get a version of the log-determinant functional (2.7) which is scale-invariant (invariant under $w \mapsto w+a, a \in \mathbb{R}$ ), and dispense with the volume-preservation condition $v(M, g)=\int_{M} e^{4 w}=v_{0}$, as follows: Let

$$
\begin{gathered}
F_{A}(w)=-\frac{1}{4} \beta_{2}\left\{\frac{1}{4} k\left(M, g_{0}, A\right) \log f_{M} e^{4(w-\bar{w})}-\int_{M}(\Delta w)^{2}-J_{0} \int_{M}|d w|^{2}\right\} \\
-\beta_{3}\left\{J_{0} \int_{M}|d w|^{2}-\frac{1}{2} \int_{M}\left(\frac{\Delta e^{w}}{e^{w}}\right)^{2}\right\},
\end{gathered}
$$


where $f_{M}=v_{0}^{-1} \int_{M}$ and $\bar{w}=f_{M} w . \quad F_{A}(w)$ is scale-invariant, and agrees with $\left(-\log |\operatorname{det} A|+\log \left|\operatorname{det} A_{0}\right|\right) / 2 l$ when $v(M, g)=v_{0}$. In fact,

$$
F_{A}(w)=-\frac{1}{4}\left(\int_{M} U_{2}[A]\right) \log \frac{v(M, g)}{v_{0}}-\frac{\log \left|\operatorname{det} A_{1}\right|-\log \left|\operatorname{det} A_{0}\right|}{2 l},
$$

where $g_{1}=e^{2 w} g_{0}$. In particular, $F_{A}(w)$ is a spectral invariant; that is, it depends only on the spectrum of $A$ in the metric $g$, since this is true of the functional determinant and the heat invariants $\int_{M} U_{i}[A]$. The volume $v\left(M, g=e^{2 w} g_{0}\right)$ is, of course, just the heat invariant $\int_{M} U_{0}[A]$, up to a positive constant multiple which depends on the leading symbol of $A$ as a universal polynomial, but not on $(M, g)$.

Theorem 2.9. Suppose $\left(M, g_{0}\right)$ and $A$ are as in Proposition 2.5, that $k\left(M, g_{0}, A\right)<32 \pi^{2}$, and that $\beta_{2}[A] \beta_{3}[A]>0$. (For example, $A$ could be the conformal Laplacian L or the square $\nabla^{2}$ of the Dirac operator.) If $c_{1} \in \mathbb{R}$ and $c_{2}>0$, then

$$
X_{c_{1}, c_{2}}[A]:=\left\{w \in C^{\infty}(M) \mid\left(\operatorname{sgn} \beta_{2}[A]\right) F_{A}(w) \leqq c_{1}, f_{M}^{f} e^{4 w}=c_{2}\right\}
$$

is uniformly bounded in the $W^{2,2}$ norm, with

$$
\|w\|_{2,2} \leqq \operatorname{const}\left(c_{1}, c_{2}, M, g_{0}, A\right), \quad \text { all } w \in X_{c_{1}, c_{2}}[A] .
$$

Proof. First assume that $\beta_{2}[\mathrm{~A}]<0$. If $w$ is not constant, we may apply the inequality $0 \leqq(\mu-v)^{2}$ with $\mu=2 \pi(w-\bar{w})$ and $v=(8 \pi)^{-1} \int(\Delta w)^{2}$ to obtain

$$
4(w-\bar{w}) \leqq \frac{32 \pi^{2}(w-\bar{w})^{2}}{\int(\Delta w)^{2}}+\frac{1}{8 \pi^{2}} \int(\Delta w)^{2} .
$$

Proposition 2.2 in the form (2.2) now implies that

$$
\log _{M} f e^{4(w-\bar{w})} \leqq \log c_{0}+\frac{1}{8 \pi^{2}} \int_{M}(\Delta w)^{2} .
$$

Thus from (2.10) we get

$$
\begin{aligned}
F_{A}(w) \leqq & -\frac{1}{4} \beta_{2}\left\{\frac{1}{4} k \log c_{0}+\left(\frac{k}{32 \pi^{2}}-1\right) \int_{M}(\Delta w)^{2}\right\} \\
& +\left(\frac{1}{4} \beta_{2}-\beta_{3}\right) J_{0} \int_{M}|d w|^{2}+\frac{1}{2} \beta_{3} \int_{M}\left(\frac{\Delta e^{w}}{e^{w}}\right)^{2},
\end{aligned}
$$

where $k=k\left(M, g_{0}, A\right)$. We recall that $a:=k / 32 \pi^{2}<1$ and rewrite the above as

$$
\begin{aligned}
-\frac{1}{4} \beta_{2}(1-a) \int_{M}(\Delta \omega)^{2}-\frac{1}{2} \beta_{3} \int_{M}\left(\frac{\Delta e^{w}}{e^{w}}\right)^{2} \leqq & \left(\frac{1}{4} \beta_{2}-\beta_{3}\right) J_{0} \int_{M}|d w|^{2} \\
& -\frac{1}{16} \beta_{2} k \log c_{0}-F_{A}(w) \\
\leqq & \left(\frac{1}{4} \beta_{2}-\beta_{3}\right) J_{0} \int_{M}|d w|^{2} \\
& +c_{1}+\operatorname{const}\left(M, g_{0}, A\right) .
\end{aligned}
$$


We expand the exponential term as

$$
\int_{M}\left(\frac{\Delta e^{w}}{e^{w}}\right)^{2}=\int_{M}\left(\Delta w-|d w|^{2}\right)^{2}=\int_{M}(\Delta w)^{2}-2 \int_{M}(\Delta w)|d w|^{2}+\int_{M}|d w|^{4},
$$

and estimate the cross term in this by

$$
\begin{aligned}
\left.2\left|\int_{M}(\Delta w)\right| d w\right|^{2} \mid & \leqq 2\left(\int_{M}(\Delta w)^{2}\right)^{1 / 2}\left(\int_{M}|d w|^{4}\right)^{1 / 2} \\
& \leqq \lambda \int_{M}(\Delta w)^{2}+\lambda^{-1} \int_{M}|d w|^{4}
\end{aligned}
$$

for any $\lambda>0$. By the sign assumptions on $\beta_{2}, \beta_{3}$, we may choose $\lambda>1$ so that

$$
b:=-\frac{1}{4} \beta_{2}(1-a)-\frac{1}{2} \beta_{3}(1-\lambda)>0 ;
$$

this leaves us with

$$
\begin{aligned}
b \int_{M}(\Delta w)^{2}-\frac{1}{2} \beta_{3}\left(1-\lambda^{-1}\right) \int_{M}|d w|^{4} \leqq & \left(\frac{1}{4} \beta_{2}-\beta_{3}\right) J_{0} \int_{M}|d w|^{2} \\
& +c_{1}+\operatorname{const}\left(M, g_{0}, A\right) .
\end{aligned}
$$

The first term on the right of this can be estimated via

$$
2 \int_{M}|d w|^{2} \leqq 2\left(\int_{M}|d w|^{4}\right)^{1 / 2} v_{0}^{1 / 2} \leqq \varepsilon \int_{M}|d w|^{4}+\varepsilon^{-1} v_{0}
$$

for any $\varepsilon>0$. We can now choose $\varepsilon=\varepsilon\left(M, g_{0}, A\right)$ so that

$$
\int_{M}(\Delta w)^{2}+\int_{M}|d w|^{4} \leqq \operatorname{const}\left(c_{1}, M, g_{0}, A\right) .
$$

To achieve a $W^{2,2}$ bound, we still need an estimate on $w$ itself to combine with our bounds on $\|\Delta w\|_{2}$ and $\|d w\|_{4}$. This comes from the fact that the normalized volume $f_{M} e^{4 w}$ is fixed at $c_{2}$. Indeed, by (2.12), $-\bar{w}+c_{2} \leqq \operatorname{const}\left(c_{1}, M, g_{0}, A\right)$; this is a bound from below on $\bar{w}$. On the other hand, $e^{4 \bar{w}} \leqq f_{M} e^{4 w}=c_{2}$ is a bound from above. The upshot is that $|\bar{w}|$ is bounded by a constant depending on $c_{1}, c_{2}, M, g_{0}$, and $A$.

The case $\beta_{2}[A]>0$ is entirely similar.

Corollary 2.10. Suppose that $\left(M, g_{0}\right)$ is as in Proposition 2.5. If $16 \pi^{2} \chi(M)$ $<32 \pi^{2}+\frac{3}{2} v_{0} c^{2}$, then

$$
X_{c_{1}, c_{2}}[L]=\left\{w \in C^{\infty}(M) \mid-F_{L}(w) \leqq c_{1}, f_{M}^{f} e^{4 w}=c_{2}\right\}
$$

is uniformly bounded in the $W^{2,2}$ norm by a constant depending on $c_{1}, c_{2}$, and $\left(M, g_{0}\right)$. If $M$ has spin structure and $16 \pi^{2} \chi(M)<32 \pi^{2}+\frac{9}{11} v_{0} c^{2}$, the same conclusion holds for

$$
X_{c_{1}, c_{2}}\left[\not^{2}\right]=\left\{w \in C^{\infty}(M) \mid F_{\nabla^{2}}(w) \leqq c_{1}, \underset{M}{f} e^{4 w}=c_{2}\right\} .
$$

Corollary 2.11. If $\left(M, g_{0}, A\right)$ is as in Proposition 2.5 and Theorem 2.9, and if $\left(\operatorname{sgn} \beta_{2}[A]\right) F_{A}(w) \leqq c_{1} \in \mathbb{R}$, then $\int_{M} J_{g}^{2} d v_{g} \leqq \operatorname{const}\left(M, g_{0}, A, c_{1}\right)$, where $6 J_{g}$ is the 
scalar curvature of the metric $g=e^{2 w} g_{0}$. In particular we have this bound when $A$ is the conformal Laplacian $L$ or the square $\not^{2}$ of the Dirac operator.

Proof. Let $u=e^{w}$. Then $d v_{g}=u^{4} d v_{0}$, where $d v_{0}=d v_{g_{0}}$, and the Yamabe equation reads

$$
\Delta u+J_{0} u=J_{g} u^{3},
$$

where the Laplacian is that of $g_{0}$. Hence

$$
\int_{M} J(g)^{2} d v_{g}=\int_{M} J_{g}^{2} u^{4} d v_{0}=\int_{M}\left(\frac{\Delta e^{w}}{e^{w}}+J_{0}\right)^{2} d v_{0},
$$

which is bounded by the argument in the proof of Theorem 2.9.

Corollary 2.12. If $\left(M, g_{0}, A\right)$ is as in Proposition 2.5 and Theorem 2.9, and if

$$
\left(\operatorname{sgn} \beta_{2}[A]\right) F_{A}(w) \leqq c_{1} \in \mathbb{R},
$$

then $\int_{M}\left|\rho_{g}\right|^{2} \leqq \operatorname{const}\left(M, g_{0}, A, c_{1}\right)$, where $\rho_{g}$ is the Ricci tensor of the metric $g=e^{2 w} g_{0}$. In particular we have this bound when $A$ is the conformal Laplacian $L$ or the square $\not^{2}$ of the Dirac operator.

Proof. By (2.8), the heat invariant $\int_{M} U_{2}[A]$ has the form

$$
\int_{M} U_{2}[A]=\int_{M}\left\{\beta_{1}|C|^{2}+\beta_{2}\left(J^{2}-|V|^{2}\right)\right\}
$$

for a general Riemannian metric in four dimensions; this is a consequence of the conformal assumption on $A$. Thus $\int_{M} U_{2}[A]$ is a linear combination of the Euler characteristic

$$
\begin{aligned}
\chi(M) & =\left(32 \pi^{2}\right)^{-1} \int_{M}\left(|C|^{2}-8|V|^{2}+8 J^{2}\right) \\
& =\left(32 \pi^{2}\right)^{-1} \int_{M}\left(|C|^{2}-8|B|^{2}+6 J^{2}\right)
\end{aligned}
$$

and $\int_{M}|C|^{2}$, which is a conformal invariant. Since $\chi(M)$ is a topological invariant, $\int_{M} U_{2}[A]$ is a conformal invariant. (This phenomenon occurs more generally [BØ1]: if $A$ is a positive integer power of a conformal covariant in dimension $2 m$ which is formally self-adjoint and has positive definite leading symbol, then $\int U_{n}[A]$ is a conformal invariant.) As a result, $\int_{M}\left|V_{g}\right|^{2} d v_{g}$, and thus

$$
\int_{M}\left|\rho_{g}\right|^{2} d v_{g}=\int_{M}\left(4\left|V_{g}\right|^{2}+8 J_{g}^{2}\right) d v_{g}
$$

has a bound depending on $M, g_{0}, A$, and $c_{1}$.

\section{Locally Symmetric Einstein 4-Manifolds, Case by Case}

In [J], G. Jensen classifies the simply connected, homogeneous Einstein manifolds of dimension 4; it turns out that all of these are symmetric, a condition that does not persist in higher dimensions. The list, grouped according to $\operatorname{sgn} J_{0}$, is:

$$
\begin{array}{ll}
J_{0}>0: & \text { (a) } \mathbb{S}^{4}, \text { (b) } \mathbb{C P}^{2}, \text { (c) } \mathbb{S}^{2} \times \mathbb{S}^{2} ; \\
J_{0}=0: & \text { (d) } \mathbb{R}^{4} ; \\
J_{0}<0: & \text { (e) } \mathbb{H}^{4}, \text { (f) } \mathbb{C} \mathbb{H}^{2}, \text { (g) } \mathbb{H}^{2} \times \mathbb{H}^{2} .
\end{array}
$$


(In each case there is an unambiguous choice of "standard" metric, up to normalization.) Here $\mathbb{H}^{n}$ is the $n$-dimensional hyperbolic space, $\mathbb{C H}^{2}$ is the complex hyperbolic space of complex dimension 2, and in cases (c) and (g), we use the same metric normalization on each factor. $(\mathrm{d}-\mathrm{g})$ are group manifolds; that is, they admit simply transitive Lie groups of isometries. The compact, locally symmetric, Einstein 4-manifolds, being quotients of simply connected symmetric spaces by cocompact deck transformation groups of isometries, are compact quotients of spaces from the above list, inheriting the standard metrics. In this section, we examine the spaces of each type from the point of view of our previous analysis; specifically, we need to identify $k\left(M, g_{0}, A\right)$, and determine whether the null space $\mathscr{N}(A)$ vanishes, as required for Proposition 2.5. In case (d), this null space never vanishes, but we show that our analysis applies nonetheless. Note that by (2.14), a compact Einstein 4-manifold has nonnegative Euler characteristic, and has Euler characteristic 0 if and only if it is flat (has vanishing Riemann tensor).

a. The four-sphere $\mathbb{S}^{4}$ has Euler characteristic 2, and its standard metric $g_{0}$ is conformally flat, so that $k\left(\mathbb{S}^{4}, g_{0}, A\right)=k\left(\mathbb{S}^{4}\right)=32 \pi^{2}$. Thus $\left(\mathbb{S}^{4}, g_{0}\right)$ is just outside the reach of our results in Sect. 2; because of this, and because of the closely related circumstance of a large conformal diffeomorphism group, one expects the logdeterminant functional here to be especially interesting. We shall explore this in detail in Sect. 4 below. By Synge's Theorem, there is only one possible quotient; this is the real projective space $\mathbb{R}^{4}$. This has Euler characteristic 1, so that $k\left(\mathbb{R} \mathbb{P}^{4}, g_{0}, A\right)=k\left(\mathbb{R} \mathbb{P}^{4}\right)=16 \pi^{2}<32 \pi^{2}$. Thus the only way our results can fail to apply is for $\mathscr{N}(A)$ to be nonzero. But the bottom eigenvalue of $L_{0}$ is $J_{0}=2$, and by the Lichnerowicz formula $\phi^{2}-\nabla^{*} \nabla=\tau / 4=3 J / 2$, the bottom eigenvalue of $\phi_{0}^{2}$ is at least 3 . As a result, $\left(\mathbb{R}^{4}, g_{0}\right)$ together with $L$ or $\not^{2}$ satisfy the hypotheses of Theorem 2.9.

b. Consider $\mathbb{C P}^{2}$, and let $g_{0}$ be the Fubini-Study metric, normalized so that the (constant) holomorphic sectional curvature is $h=4$; this is the usual normalization on any $\mathbb{C P}^{m}[$ Bes, 2.59]. By [Bes,11.5 and 11.12], the volume and scalar curvature of (any normalization of) $g_{0}$ are related by

$$
v_{0} \tau_{0}^{2}=288 \pi^{2} \text {. }
$$

Since $\tau_{0}=6 h, J_{0}=4$, and $v_{0}=\pi^{2} / 2$. Since $\chi\left(\mathbb{C P}^{2}\right)=3,(2.14)$ gives $c^{2}=96$. Thus

$$
k\left(\mathbb{C P}^{2}, g_{0}, A\right)=48 \pi^{2}\left(4 \frac{\beta_{1}[A]}{\beta_{2}[A]}-1\right),
$$

so that by Remark 2.8, the hypotheses of Theorem 2.9 are satisfied provided $\beta_{1}[A] / \beta_{2}[A]<5 / 12$ and $\mathscr{N}(A)=0$. By (2.9), the inequality constraint is no problem for $L$. Since $\mathbb{C I P}^{2}$ does not have spin structure [Bes, 6.72], there is no Dirac operator. (Being a complex manifold, $\mathbb{C P}^{2}$ is orientable, so there is no Pin(4) structure either.) And, arguing as in Sect. 4.a above, the bottom eigenvalue of $L$ is 4 , so that the null space constraint is no problem. By Synge's Theorem, $\mathbb{C P}^{2}$ is a double cover of any metric quotient $\left(M, g_{0}\right)$ it might have, but since $\left.\chi(\mathbb{C P})^{2}\right)$ is odd, there is no such quotient.

c. Let $M=\mathbb{S}^{2} \times \mathbb{S}^{2}$, and let $g_{0}$ be the product of standard $\mathbb{S}^{2}$ metrics. Then $\chi(M)=\chi\left(\mathbb{S}^{2}\right)^{2}=4, J_{0}=2 / 3$, and $v_{0}=16 \pi^{2}$. As a consequence, $c^{2}=16 / 3$ by (2.14). (That this space is conformally curved is perhaps contrary to naive expectation; we remark that the standard pseudo-Riemannian metric of signature (2.2) on 
$\mathbb{S}^{2} \times \mathbb{S}^{2}$ is conformally flat.) As a consequence of the conformal curvature, $\beta_{1} / \beta_{2}$ comes into play, and

$$
k\left(\mathbb{S}^{2} \times \mathbb{S}^{2}, g_{0}, A\right)=32 \pi^{2}\left(\frac{2}{3}+\frac{32}{3} \frac{\beta_{1}[A]}{\beta_{2}[A]}\right) .
$$

Thus the analysis of Sect. 2 applies as long as $A_{0}$ has no zero eigenvalues and $32 \beta_{1}[A] / \beta_{2}[A]<1$. In particular, this covers the cases of the conformal Laplacian $L$ and the square $\not^{2}$ of the Dirac operator: arguing as in Sect. 3.a, the bottom eigenvalue of $L_{0}$ is $J_{0}=2 / 3$, and the bottom eigenvalue of $\phi_{0}^{2}$ is at least 1 . In each case, $\beta_{1}[A] / \beta_{2}[A]<0$, so the hypotheses of Theorem 2.9 are satisfied. If $M$ is a compact quotient of $\mathbb{S}^{2} \times \mathbb{S}^{2}$, then $\chi(M)<4$, and the bottom eigenvalue of a natural differential operator with positive definite leading symbol can only increase upon passage from the covering to the covered space (depending on whether or not the bottom eigenfunctions or eigensections descend to the quotient). Thus any $A$ satisfying the hypotheses of Theorem 2.9 on $\mathbb{S}^{2} \times \mathbb{S}^{2}$ satisfies them on $M$ also; in particular, this is true for $L$ and $\phi^{2}$.

d. Suppose $\left(M, g_{0}\right)$ is covered by $\mathbb{R}^{4}$, with the standard $\mathbb{R}^{4}$ metric as the pullback of $g_{0}$. Here $\chi(M)=c^{2}=J_{0}=0$, so $k\left(M, g_{0}, A\right)=k(M)=0<32 \pi^{2}$. A complication arises from the fact that all natural differential operators $A$ on $\mathbb{R}^{4}$ have 0 as an eigenvalue, so that one of the hypotheses of Proposition 2.5 may be violated if eigensections descend to $M$. The zero eigenfunctions or eigensections on $\mathbb{R}^{4}$, however, can be written down explicitly: each tensor-spinor bundle over $\mathbb{R}^{n}$ has a standard trivialization. A tensor-spinor is annihilated by an elliptic natural differential operator $A$ exactly when it has constant components in this trivialization. Let $\mathscr{N}(A)_{M}$ be the space of such constant sections that descend to $M$, and let $q=\operatorname{dim} \mathscr{N}(A)_{M}$. By an argument in [BØ3, Sect. 3.b], the effect on the scale invariant log-determinant functional is to change $F_{A}(w)$ to

$$
\tilde{F}_{A}(w)=F_{A}(w)+\frac{q}{4} \log \frac{v_{1}^{1 / 4} v_{r / 4}^{-1 / r}}{v_{0}^{1 / r-1 / 4}},
$$

where $v_{\varepsilon}=\int_{M} e^{4 \varepsilon w}$ is the volume of $e^{2 \varepsilon w} g_{0}$, and $\cdot r$ is the order of the conformal covariant of which $A$ is a power. (For example, if $A=L$, then $r=2$; if $A=\not^{2}$, then $r=1$.) If $r=4$, then $\widetilde{F}_{A}(w)=F_{A}(w)$, and all is well. If $r<4$, the convexity of the exponential function gives

$$
v_{1}^{r / 4} \leqq v_{r / 4} \leqq \max \left(v_{0}, v_{1}\right) .
$$

Thus the proof of Theorem 2.9 (without the assumption $\mathscr{N}(A)=0$. and replacing $F_{A}$ by $\left.\widetilde{F}_{A}\right)$ proceeds as before, until we get to $(2.13)$, where we need only replace "const $\left(M, g_{0}, A\right)$ " on the right with "const $\left(M, g_{0}, A, c_{2}\right)$." The conclusion of the theorem is valid without modification.

e. Let $M$ be a compact quotient of $\mathbb{H}^{4}$, inheriting the standard metric $g_{0}$ of constant sectional curvature -1 . Then $\left(M, g_{0}\right)$ is a conformally flat Einstein manifold; by (2.14), $\chi(M)=3 v_{0} / 4 \pi^{2} ; \quad$ in particular, $\quad \chi(M)>0$. Thus $k\left(M, g_{0}, A\right)=k(M)=16 \pi^{2} \chi(M)$, and the constraint $k(M)<32 \pi^{2}$ becomes $\chi(M)<2$; as a result, we need $\chi(M)=1$ (so that $v_{0}=4 \pi^{2} / 3$ ). It is apparently very difficult to say anything elementary about the constraint $\mathscr{N}(A)=0$, so in working with this example, we add it as a side condition. 
f. The usual Bergmann metric $g_{0}$ on any $\mathbb{C} \mathbb{H}^{m}$ has constant holomorphic sectional curvature $h=-4$ [Bes, 2.61]; this corresponds to $J_{0}=-4$. By duality, the value of $c^{2}$ on $\mathbb{C I H}^{m}$ is the same as that for $\mathbb{C P}^{m}$; in the case $m=2$, that value is $c^{2}=96$ by the above. If $M$ is a compact quotient with volume $v_{0}$, then $\chi(M)=6 v_{0} / \pi^{2}$ by (2.14); in particular, $\chi(M)>0$ and $v_{0} \geqq \pi^{2} / 6$. This yields

$$
k\left(M, g_{0}, A\right)=16 \pi^{2} \chi(M)\left(4 \frac{\beta_{1}[A]}{\beta_{2}[A]}+\frac{1}{2}\right) .
$$

Thus we need $\chi(M)\left(1+8 \beta_{1}[A] / \beta_{2}[A]\right)<4$ to have $k\left(M, g_{0}, A\right)<32 \pi^{2}$. In particular, by (2.9), $k\left(M, g_{0}, L\right)<32 \pi^{2}$ regardless of the value of $\chi(M)$, but to have $k\left(M, g_{0}, \not^{2}\right)<32 \pi^{2}$, we need $\chi(M)<11$. Again, it is very difficult to treat the constraint $\mathscr{N}(A)=0$, so we regard it as a side condition.

g. A compact quotient $M$ of $\mathbb{H}^{2} \times \mathbb{H}^{2}$, endowed with the product $g_{0}$ of FubiniStudy metrics of constant sectional curvature -1 , has $J_{0}=-2 / 3$. Duality with the $\mathbb{S}^{2} \times \mathbb{S}^{2}$ situation gives $c^{2}=16 / 3$, and (2.14) gives $\chi(M)=v_{0} / 4 \pi^{2}$. By Remark 2.8 ,

$$
k\left(M, g_{0}, A\right)=32 \pi^{2} \chi(M)\left\{\frac{1}{6}+\frac{8}{3} \frac{\beta_{1}[A]}{\beta_{2}[A]}\right\} .
$$

Thus the constraint $k\left(M, g_{0}, A\right)<32 \pi^{2}$ reads $\chi(M)\left(1+16 \beta_{1}[A] / \beta_{2}[A]\right)<6$. In view of (2.9), this always holds for $L$ and, when $M$ has spin structure, $\not^{2}$ (regardless of the value of $\chi(M)$ ). Again we view the constraint $\mathscr{N}(A)=0$ as a side condition.

\section{Boundedness in the Standard Conformal Class on the Four-Sphere}

In this section, we show that a $W^{2,2}$-boundedness result holds in the conformal class $C_{+}^{\infty}\left(\mathbb{S}^{4}\right) g_{0}=\left\{e^{2 w} g_{0} \mid w \in C^{\infty}\left(\mathbb{S}^{4}\right)\right\}$ of the standard metric $g_{0}$ on $\mathbb{S}^{4}$, even though $k\left(M, g_{0}, A\right)$ is exactly equal to the borderline value $32 \pi^{2}$ in this case. Since the conformal group of $\mathbb{S}^{4}$ is not compact, the conclusion of the theorem has to be modified. We do this by specifying a "centeredness" property that is enjoyed by at least one conformal transform of any $g \in C_{+}^{\infty}\left(\mathbb{S}^{4}\right) g_{0}$, and then showing that a set of centered metrics with the appropriate spectral invariants bounded is bounded in $W^{2,2}$ norm.

First note that if $\varphi$ is a conformal transformation on a Riemannian $n$-manifold $(M, g)$, that is, $\varphi^{*} g=\Omega_{\varphi}^{2} g$ for some $0<\Omega_{\varphi} \in C^{\infty}(M)$, then $\Omega_{\varphi}$ is necessarily $|J \varphi|^{1 / n}$, where $J \varphi$ is the Jacobian determinant. If $w \in C^{\infty}(M)$, then

$$
\varphi^{*}\left(e^{2 w} g\right)=e^{2 w \circ \varphi} \Omega_{\varphi}^{2} g=\exp (2 w \circ \varphi+2(\log |J \varphi|) / n) g .
$$

Thus (specializing to $\left(\mathbb{S}^{4}, g_{0}\right)$ ), we would like to identify elements of the class

$$
[w]=\left\{T_{\varphi} w \mid \varphi \in \mathscr{C}\left(\mathbb{S}^{4}, g_{0}\right)\right\},
$$

where $T_{\varphi} w=w \circ \varphi+(\log |J \varphi|) / 4$. We denote the corresponding equivalence relation by $\sim: w \sim w^{\prime}$ if and only if $[w]=\left[w^{\prime}\right]$. Our result is:

Theorem 4.1. Let $c_{1} \in \mathbb{R}$ and $0<c_{2} \in \mathbb{R}$. On $\left(\mathbb{R}^{4}, g_{0}\right)$, with $A$ as in Proposition 2.5, let

$$
W=W_{c_{1}, c_{2}}:=\left\{w \in C^{\infty}\left(\mathbb{S}^{4}\right) \mid F_{A}(w)=c_{1}, \underset{\mathbb{S}^{4}}{f} e^{4 w}=c_{2}\right\} .
$$


( $f_{\mathbb{S}^{4}}$ is the normalized integral in the standard metric.) Then $W$ is bounded in $W^{2,2} / \sim$, in the sense that for each $w \in W$, there is a $\tilde{w} \in[w]$ such that $\{\tilde{w} \mid w \in W\}$ is uniformly bounded in the $W^{2,2}$ norm, with

$$
\|\tilde{w}\|_{2,2} \leqq \operatorname{const}\left(c_{1}, c_{2}, A\right) .
$$

The proof of this theorem which we shall present below follows the strategy used in [On, CY, OPS1] to prove the corresponding result for $\mathbb{S}^{2}$.

Let

$$
\mathscr{S}_{0}=\left\{w \in W^{2,1}\left(\mathbb{S}^{4}\right) \mid \underset{\mathbb{S}^{4}}{f} e^{4 w} x_{j}=0, j=1,2,3,4,5\right\},
$$

where the $x_{j}$ are homogeneous coordinates on $\mathbb{S}^{4}$. A basic fact we shall use is:

Lemma 4.2. Given $w \in W^{2,1}\left(\mathbb{S}^{4}\right)$, there is some $\varphi \in \mathscr{C}\left(\mathbb{S}^{4}, g_{0}\right)$ with $T_{\varphi} w \in \mathscr{S}_{0}$.

Proof. The identity component of the conformal transformation group of $\mathbb{S}^{n}$ is $G=S O_{0}(n+1,1) ; K=S O(n+1)$ is the maximal compact subgroup. The "noncompact part" of $G$, the symmetric space $G / K$, can be naturally identified with the ball $\mathbb{B}^{n+1}$; in fact, with the metric induced by the Killing form of $G$, this is one of the standard realizations of the hyperbolic space $\mathbb{H}^{n+1}$. The lemma is a simple consequence of the fixed point theorem; a complete proof can be found in [CY].

The following is an analogue of the Adams inequality (which involves $\left\|\Delta^{n / 2} w\right\|$ in dimension $n$ ) with a proof analogous to that of a result of Aubin [Au1] involving $\|\nabla w\|_{n}$ on $\mathbb{S}^{n}$.

Lemma 4.3. If $w \in \mathscr{S}_{0}$, then for any $\varepsilon>0$, there exist constants $C_{1}(\varepsilon)$ and $C_{2}(\varepsilon)$ with

$$
\begin{aligned}
\log \underset{\mathbb{S}^{4}}{f} e^{4(w-\bar{w})} & \leqq C_{1}(\varepsilon)+\frac{1}{2}\left(\frac{1}{8 \pi^{2}}+\varepsilon\right) \underset{\mathbb{S}^{4}}{f}(\Delta w)^{2}+C_{2}(\varepsilon) \underset{\mathbb{S}^{4}}{f}|d w|^{2} \\
& \leqq C_{1}(\varepsilon)+\left(\frac{1}{6}+\varepsilon\right)_{\mathbb{S}^{4}}(\Delta w)^{2}+C_{2}(\varepsilon) \underset{\mathbb{S}^{4}}{f}|d w|^{2} .
\end{aligned}
$$

Remark 4.4. (4.1) should be compared to (2.12). It is a consequence of Theorem 5.2 below that if $w=\frac{1}{4} \log |J \varphi|$ for $\varphi \in \mathscr{C}\left(\mathbb{S}^{4}, g_{0}\right)$, so that $\varphi^{*} g_{0}=e^{4 w} g_{0}$, then $(2.12)$ becomes an equality with $c_{0}=0$ on $\mathbb{S}^{4}$. Thus the coefficient $1 / 8 \pi^{2}$ in (2.12) cannot be improved.

Remark 4.5. Lemma 4.3 can be generalized to $\mathbb{S}^{n}$, with $\left\|\Delta^{n / 2} w\right\|_{2}$ as the dominant term on the right side of the analogue of (4.1). For the sake of simplicity, we present only the $\mathbb{S}^{4}$ version.

Proof of Lemma 4.3. We may assume that $\int_{\mathbb{S}^{4}} e^{4 w}=v_{0}=8 \pi^{2} / 3$, since the scaleinvariance of each term in (4.1) allows us to shift a given $w \in \mathscr{S}_{0}$ by a suitable constant. (4.1) is now equivalent to

$$
0 \leqq 4 \bar{w}+C_{1}(\varepsilon)+\left(\frac{1}{16 \pi^{2}}+\varepsilon\right) \int_{\mathbb{S}^{4}}(\Delta w)^{2}+C_{2}(\varepsilon) \underset{\mathbb{S}^{4}}{f}|d w|^{2} .
$$


To establish (4.2), cover $\mathbb{S}^{4}$ by ten cap-shaped congruent domains, each centered at one of the poles (points at which $\left|x_{i}\right|=1$ for some $i$, and $x_{j}=0$ for $j \neq i$ ). Rotating axes if necessary, we may assume that

$$
\int_{\Omega_{\delta_{1}}} e^{4 w} \geqq \frac{v_{0}}{10}, \quad \Omega_{\delta_{1}}=\left\{x \in \mathbb{S}^{4} \mid \delta_{1} \leqq x_{5} \leqq 1\right\},
$$

for some $\delta_{1} \in(0,1)$ which is independent of $w$. (In fact, we are guaranteed this for any $\delta_{1}>1 / \sqrt{5}$.) Since $\int_{\mathbb{S}^{4}} e^{4 w} x_{5}=0$, an elementary computation shows that if we choose $\delta_{2} \leqq \delta_{1} / 19$, then

$$
\int_{\tilde{\Omega}_{\delta_{2}}} e^{4 w} \geqq \delta_{2} v_{0}, \quad \tilde{\Omega}_{\delta_{2}}=\left\{x \in \mathbb{S}^{4} \mid-1 \leqq x_{5} \leqq-\delta_{2}\right\} .
$$

We now choose a cutoff function $\varphi_{1}$, where $\varphi_{1} \equiv 1$ on $\Omega_{\delta_{1}}, 0 \leqq \varphi_{1} \leqq 1$, and $\varphi_{1} \equiv 0$ off $\Omega_{\delta_{1 / 2}}$. Let $\varphi_{2}$ be the similarly-defined cutoff function for $\left(\tilde{\Omega}_{\delta_{2}}, \widetilde{\Omega}_{\delta_{2} / 2}\right)$. Note that the $\varphi_{k}$ can be taken to be independent of $w$, since the $\delta_{k}$ are. Applying (2.12) to the functions $(w-\bar{w}) \varphi_{k}$, we obtain

$$
\begin{aligned}
\frac{v_{0}}{10} & \leqq \int_{\Omega_{\delta_{1}}} e^{4 w} \leqq e^{4 \bar{w}} \int_{\mathbb{S}^{4}} e^{4(w-\bar{w}) \varphi_{1}} \\
& \leqq e^{4 \bar{w}} v_{0} c_{0} \exp \left\{4 \underset{\mathbb{S}^{4}}{f}(w-\bar{w}) \varphi_{1}+\left(8 \pi^{2}\right)^{-1} \int_{\mathbb{S}^{4}}\left(\Delta\left[(w-\bar{w}) \varphi_{1}\right]\right)^{2}\right\}
\end{aligned}
$$

and similarly,

$$
\begin{aligned}
\delta_{2} v_{0} & \leqq \int_{\tilde{\Omega}_{\delta_{2}}} e^{4 w} \\
& \leqq e^{4 \bar{w}} v_{0} c_{0} \exp \left\{4 \underset{\mathbb{S}^{4}}{f}(w-\bar{w}) \varphi_{2}+\left(8 \pi^{2}\right)^{-1} \int_{\mathbb{S}^{4}}\left(\Delta\left[(w-\bar{w}) \varphi_{2}\right]\right)^{2}\right\} .
\end{aligned}
$$

We now observe that

$$
\begin{aligned}
\int_{\mathbb{S}^{4}}\left(\Delta\left[(w-\bar{w}) \varphi_{1}\right]\right)^{2}= & \int_{\mathbb{S}^{4}}(\Delta w)^{2} \varphi_{1}^{2}+4 \int_{\mathbb{S}^{4}}\left\langle d w, d \varphi_{1}\right\rangle^{2}+\int_{\mathbb{S}^{4}}\left(\Delta \varphi_{1}\right)^{2}(w-\bar{w})^{2} \\
& -4 \int_{\mathbb{S}^{4}}(\Delta w) \varphi_{1}\left\langle d w, d \varphi_{1}\right\rangle+2 \int_{\mathbb{S}^{4}}(\Delta w) \varphi_{1}(w-\bar{w}) \Delta \varphi_{1} \\
& -4 \int_{\mathbb{S}^{4}}\left\langle d w, d \varphi_{1}\right\rangle(w-\bar{w}) \Delta \varphi_{1} \\
\leqq & \int_{\Omega_{\delta_{1} / 2}}(\Delta w)^{2}+\varepsilon \int_{\mathbb{S}^{4}}(\Delta w)^{2}+C\left(\varepsilon, \delta_{1}\right) \int_{\mathbb{S}^{4}}|d w|^{2}
\end{aligned}
$$

for some suitable constant $C\left(\varepsilon, \delta_{1}\right)$. Similarly,

$$
\int_{\mathbb{S}^{4}}\left(\Delta\left[(w-\bar{w}) \varphi_{2}\right]\right)^{2} \leqq \int_{\Omega_{\delta_{2} / 2}}(\Delta w)^{2}+\varepsilon \int_{\mathbb{S}^{4}}(\Delta w)^{2}+C\left(\delta_{2}, \varepsilon\right) \int_{\mathbb{S}^{4}}|d w|^{2}
$$

Because the bottom nonzero eigenvalue of the Laplacian on $\mathbb{S}^{4}$ is 4 , we also have

$$
\begin{aligned}
f_{\mathbb{S}^{4}}(w-\bar{w}) \varphi_{k} & \leqq \underset{\mathbb{S}^{4}}{f}|w-\bar{w}| \leqq\left(\underset{\mathbb{S}^{4}}{f}(w-\bar{w})^{2}\right)^{1 / 2} \\
& \leqq \frac{1}{2}\left(\underset{\mathbb{S}^{4}}{f}|d w|^{2}\right)^{1 / 2} \leqq \frac{1}{16}+\underset{\mathbb{S}^{4}}{f}|d w|^{2}
\end{aligned}
$$


Inserting estimates $(4.5,4.7)$ into $(4.3)$, we obtain

$$
\frac{1}{10} \leqq e^{4 \bar{w}} c_{0} \exp \left\{1+\left(8 \pi^{2}\right)^{-1} \int_{\Omega_{\delta_{1} / 2}}(\Delta w)^{2}+\varepsilon \int_{\mathbb{S}^{4}}(\Delta w)^{2}+C\left(\varepsilon, \delta_{1}\right) \int_{\mathbb{S}^{4}}|d w|^{2}\right\} .
$$

Similarly, from $(4.6,4.7)$,

$$
\delta_{2} \leqq e^{4 \bar{w}} c_{0} \exp \left\{1+\left(8 \pi^{2}\right)^{-1} \int_{\tilde{\Omega}_{\delta_{2} / 2}}(\Delta w)^{2}+\varepsilon \int_{\mathbb{S}^{4}}(\Delta w)^{2}+C\left(\varepsilon, \delta_{2}\right) \int_{\mathbb{S}^{4}}|d w|^{2}\right\} .
$$

We now multiply (4.8)and (4.9) and take the square root on both sides, noting that $\Omega_{\delta_{1 / 2}}$ and $\widetilde{\Omega}_{\delta_{2} / 2}$ are disjoint regions in $\mathbb{S}^{4}$, to obtain:

$$
\sqrt{\delta_{2} / 10} \leqq e^{4 \bar{w}} c_{0} \exp \left\{1+\left(\frac{1}{16 \pi^{2}}+\varepsilon\right) \int_{\mathbb{S}^{4}}(\Delta w)^{2}+C\left(\varepsilon, \delta_{1}, \delta_{2}\right) \int_{\mathbb{S}^{4}}|d w|^{2}\right\} .
$$

Since we can make concrete choices of the $\delta_{k}$ (any $\delta_{1}>1 / \sqrt{5}$, and then any $\left.\delta_{2} \leqq \delta_{1} / 19\right)$, (4.2) follows.

Proof of Theorem 4.1. Given $w \in C^{\infty}\left(\mathbb{S}^{4}\right)$, we may apply Lemma 4.2 to transform to $\tilde{w} \in[w] \cap \mathscr{S}_{0}$. Assuming without loss of generality that $w \in \mathscr{S}_{0}$, the strategy used in the Proof of Theorem 2.9 works if we use Lemma 4.3 in place of (2.12).

\section{Extremals of the Log-Determinant for the Conformal Laplacian and for the Square of the Dirac Operator on $\mathbb{S}^{4}$}

Recall the Polyakov formula [P1-2] for the determinant of the ordinary Laplacian in dimension two: let $\left(M, g_{0}\right)$ be a two-dimensional, compact, Riemannian manifold without boundary. Suppose $g_{0}$ has constant scalar curvature $\tau_{0}$. Let $\Delta_{0}$ be the Laplacian in the metric $g_{0}$. Then $\Delta_{w}=e^{-2 w} \Delta_{0}$ is the Laplacian in the metric $g_{w}=e^{2 w} g_{0}$, and

$$
-\log \operatorname{det} \Delta_{w}+\log \operatorname{det} \Delta_{0}=\frac{1}{3}\left(\underset{M}{f}|d w|^{2}+\tau_{0} \underset{M}{f} w\right)
$$

for all $w \in C^{\infty}(M)$ with $f_{M} e^{2 w}=1$ (that is, conformal metrics $g_{w}$ with metrics with $\left.\operatorname{vol}\left(g_{w}\right)=\operatorname{vol}\left(g_{0}\right)\right)$. Here all integrals are in the $g_{0}$-Riemannian measure, and $f_{M}=v_{0}^{-1} \int_{M}$. Specializing to $\mathbb{S}^{2}$ with its standard metric $g_{0}$ and estimating the terms on the right, one gets:

Proposition 5.1. $[\mathrm{OV}, \mathrm{On}]$ On $\left(\mathbb{S}^{2}, g_{0}\right)$,

$$
\log \frac{\operatorname{det} \Delta_{0}}{\operatorname{det} \Delta_{w}}=\frac{1}{3} S(w)
$$

where $S(w)=f_{\mathbb{S}^{2}}|d w|^{2}+2 f_{\mathbb{S}^{2}} w$, provided $f_{\mathbb{S}^{2}} e^{2 w}=1$. Furthermore, we have $S(w) \geqq 0$, with $S(w)=0$ if and only if $g_{w}=\varphi^{*} g_{0}$ for some $\varphi$ in the Möbius transformation group of $\mathbb{S}^{2}$.

The significance of this theorem is as follows: let $\operatorname{Diff}\left(\mathbb{S}^{2}\right)$ be the full diffeomorphism group of $\mathbb{S}^{2}$. Given any Riemannian metric $h$ on $\mathbb{S}^{2}$, there is a $\psi \in \operatorname{Diff}\left(\mathbb{S}^{2}\right)$ 
with $\psi^{*} h$ in the conformal class of $g_{0}$; that is, $\psi^{*} h=e^{2 w} g_{0}$ for some $w \in C^{\infty}(M)$. Diffeomorphisms preserve the spectra of natural operators like the Laplacian; thus a fortiori they preserve spectral invariants like the log-determinant of the Laplacian. As a result, the standard metric $g_{0}$ on $\mathbb{S}^{2}$ attains the minimum of the log-determinant functional on the space of all metrics, and any other metric $h$ attaining the minimum is diffeomorphic to $g_{0}$, in the sense that there exists $\xi \in \operatorname{Diff}\left(\mathbb{S}^{2}\right)$ with $\xi^{*} h=g_{0}$. Proposition 5.1 has been generalized by Osgood, Phillips, and Sarnak [OPS1], who give a complete description of the minima of the log-determinant functional for all compact two-manifolds.

The Branson-Ørsted formula, Proposition 2.5, is a four-dimensional analogue of the Polyakov formula, generalized to cover any operator with reasonable conformal properties; in particular, the conformal Laplacian and the square of the Dirac operator. We would like an analogue of the result on extremals of the logdeterminant on $\mathbb{S}^{4}$. Of course, the property that all metrics on $\mathbb{S}^{2}$ can be gotten from the standard one by making a conformal change and then applying a diffeomorphism does not carry over to higher dimensions. With the aid of sharp inequalities recently derived by W. Beckner, however, we have obtained a full analogue of the $\mathbb{S}^{2}$ result, working in the conformal class of the standard metric on $\mathbb{S}^{4}$.

Recall from $(2.10,2.11)$ that on $\left(\mathbb{S}^{4}, g_{0}\right)$, under the assumption $f_{\mathbb{S}^{4}} e^{4 w}=1$, with $A$ as in Proposition 2.5, our log-determinant functional is

$$
\begin{aligned}
\tilde{F}_{A}(w)= & \left(8 \pi^{2}\right)^{-1}(2 l)^{-1} \log \frac{\operatorname{det} A_{0}}{\operatorname{det} A_{w}} \\
= & -\frac{1}{4} \beta_{2}\left\{\log _{\mathbb{S}^{4}}^{f} e^{4(w-\bar{w})}-\frac{1}{3} f_{\mathbb{S}^{4}}(\Delta w)^{2}-\frac{2}{3} f_{\mathbb{S}^{4}}|d w|^{2}\right\} \\
& -\beta_{3}\left\{\frac{2}{3} f_{\mathbb{S}^{4}}|d w|^{2}-\frac{1}{6} f_{\mathbb{S}^{4}}\left(\frac{\Delta e^{w}}{e^{w}}\right)^{2}\right\},
\end{aligned}
$$

where as usual, $A_{w}$ is the operator $A$ evaluated in the metric $e^{2 w} g_{0}$.

Theorem 5.2. Suppose that $A$ is as in Proposition 2.5, and that $\beta_{2}[A] \beta_{3}[A]>0$. Let $\Phi_{A}(w)=\left(\operatorname{sgn} \beta_{2}[A]\right) \tilde{F}(w)$ on $\left(\mathbb{S}^{4}, g_{0}\right)$. Then $\Phi_{A}(w) \geqq 0$, with $\Phi_{A}(w)=0$ if and only if $e^{2 w} g_{0}=\varphi^{*}\left(g_{0}\right)$ for some $\varphi \in \mathscr{C}\left(\mathbb{S}^{4}, g_{0}\right)$, the conformal transformation group of $\mathbb{S}^{4}$; that is, when $e^{2 w} g_{0}$ is isometric to $g_{0}$.

Our proof of this theorem uses the following sharp inequality due to Beckner [Bec1]; this may be interpreted as a linearized, or logarithmic form of Adams' inequality (compare (2.12) to (5.3) below).

Lemma 5.3. [Bec1] If $f \in C^{\infty}\left(\mathbb{S}^{n}\right)$ has an expansion $\sum_{k=0}^{\infty} Y_{k}$ in spherical harmonics, then

$$
\log \underset{\mathbb{S}^{n}}{f} e^{f-\bar{f}} \leqq \frac{1}{2 n} \sum_{k=1}^{\infty} B(n, k) \underset{\mathbb{S}^{n}}{f}\left|Y_{k}\right|^{2},
$$

where $B(n, k)=\Gamma(n+k) /(\Gamma(n) \Gamma(k))$. Equality holds if and only if $e^{2 f / n} g_{0}=\varphi^{*} g_{0}$ for some $\varphi$ in the conformal transformation group of the standard sphere $\left(\mathbb{S}^{n}, g_{0}\right)$. 
Restricting our attention to the special case $n=4$, we have $B(4, k)=$ $k(k+1)(k+2)(k+3) / 6$. Since the eigenvalue of $\Delta$ on the space of $k^{\mathrm{th}}$-order spherical harmonics is $k(k+3)$, we may rewrite (5.2) as

$$
\begin{aligned}
\log \underset{\mathbb{S}^{4}}{f} e^{4(w-\bar{w})} & \leqq \frac{1}{3} \underset{\mathbb{S}^{4}}{f} w \Delta(\Delta+2) w \\
& =\frac{1}{3}\left(\underset{\mathbb{S}^{4}}{f}(\Delta w)^{2}+2 \underset{\mathbb{S}^{4}}{f}|d w|^{2}\right) .
\end{aligned}
$$

Proof of Theorem 5.2. Let $I$ be the coefficient of $-\frac{1}{4} \beta_{2}$ in (5.1), and let $I I$ be the coefficient of $-\beta_{3}$. By Lemma 5.3 in the form (5.3), $I \leqq 0$, with equality if and only if $e^{2 w} g_{0}=\varphi^{*} g_{0}$ for some $\varphi \in \mathscr{C}\left(\mathbb{S}^{4}, g_{0}\right)$. It now suffices to prove a similar statement about $I I$; this is the content of the following lemma.

Lemma 5.4. If $w \in C^{\infty}\left(\mathbb{S}^{4}\right)$,

$$
-6 I I=\underset{\mathbb{S}^{4}}{f}\left(\frac{\Delta e^{w}}{e^{w}}\right)^{2}-4 \underset{\mathbb{S}^{4}}{f}|d w|^{2} \geqq 0,
$$

with equality if and only if $e^{2 w} g_{0}=\varphi^{*} g_{0}$ for some $\varphi \in \mathscr{C}\left(\mathbb{S}^{4}, g_{0}\right)$.

Proof. Though we shall present a geometric proof, this inequality can be proved by purely analytic methods [Bec2]. Let $g_{w}=e^{2 w} g_{0}$ be a conformal metric on $\mathbb{S}^{4}$, and let $6 J_{0}=12$ and $6 J_{w}$ be the scalar curvatures of $g_{0}$ and $g_{w}$ respectively. By the Yamabe equation,

$$
(\Delta+2) e^{w}=J_{w} e^{3 w},
$$

so

$$
\frac{\Delta e^{w}}{e^{w}}=-2+J_{w} e^{2 w}
$$

On the other hand,

$$
\frac{\Delta e^{w}}{e^{w}}=\Delta w-|d w|^{2}
$$

so that

$$
\begin{aligned}
\underset{\mathbb{S}^{4}}{f}\left(\frac{\Delta e^{w}}{e^{w}}\right)^{2}-4 \underset{\mathbb{S}^{4}}{f}|d w|^{2} & =\underset{\mathbb{S}^{4}}{f}\left\{4-4 J_{w} e^{2 w}+J_{w}^{2} e^{4 w}+4\left(-2+J_{w} e^{2 w}\right)\right\} \\
& =-4+\underset{\mathbb{S}^{4}}{f} J_{w}^{2} e^{4 w}
\end{aligned}
$$

Now recall the Yamabe functional

$$
\begin{aligned}
Y\left(\mathbb{S}^{4}, g_{0}, w\right) & =\frac{f_{\mathbb{S}^{4}}\left|d\left(e^{w}\right)\right|^{2}+J_{0} f_{\mathbb{S}^{4}} e^{2 w}}{\left(f_{\mathbb{S}^{4}} e^{4 w}\right)^{1 / 2}} \\
& =\frac{f_{\mathbb{S}^{4}} J_{w} e^{4 w}}{\left(f_{\mathbb{S}^{4}} e^{4 w}\right)^{1 / 2}},
\end{aligned}
$$

and the corresponding sharp Sobolev inequality, which says that the infimum of $Y\left(\mathbb{S}^{4}, g_{0}, w\right)$ is attained exactly when $e^{2 w} g_{0}=\varphi^{*} g_{0}, \varphi \in \mathscr{C}\left(\mathbb{S}, g_{0}\right)$. In particular, 
the infimum is 2 . All functionals we treat are scale-invariant, so we may work under the assumption $f_{\mathbb{S}^{4}} e^{4 w}=1$ here too. (5.4) and (5.5) give

$$
-6 I I=-4+f_{\mathbb{S}^{4}} J_{w}^{2} e^{4 w} \geqq-4+\left(\underset{\mathbb{S}^{4}}{f} J_{w} e^{4 w}\right)^{2}=Y\left(\mathbb{S}^{4}, g_{0}, w\right)^{2}-4 .
$$

This completes the proof of Lemma 5.4, and thus of Theorem 5.2.

Corollary 5.5. On $\mathbb{S}^{4}$, in the conformal class of the standard metric,

$$
\log \frac{\operatorname{det} L_{0}}{\operatorname{det} L_{w}} \leqq 0, \quad \log \frac{\operatorname{det} \phi_{0}^{2}}{\operatorname{det} \phi_{w}^{2}} \geqq 0 .
$$

In each case, equality holds if and only if $e^{4 w} g_{0}=\varphi^{*} g_{0}$ for some $\varphi \in \mathscr{C}\left(\mathbb{S}^{4}, g_{0}\right)$.

Remark 5.6. Theorem 5.2 shows that the splitting of the Branson-Ørsted formula (2.7) into $\beta_{2}$ and $\beta_{3}$ contributions is quite natural. Since we have two operators with different $\beta_{3} / \beta_{2}$, it also shows that $I$ and $I I$ are separately invariant under the conformal transformation group $\mathscr{C}\left(\mathbb{S}^{4}, g_{0}\right)$; we have also verified this by direct computation.

Remark 5.7. In view of the Beckner inequality (5.1), which is valid on $\mathbb{S}^{n}$ for any $n$, it is natural to speculate that a similar result should be true for all even $n$. (For odd $n$, the determinant of an operator of our type is conformally invariant.) In fact, working jointly with $\mathrm{W}$. Beckner, we have obtained some preliminary results in this program.

\section{References}

[Ad] Adams, D.R.: A sharp inequality of J. Moser for higher order derivatives. Ann. Math. 128, 385-398 (1988)

[Au1] Aubin, T.: Fonction de Green et valeurs propres du Laplacien. J. Math. Pures Appl. 53, 347-371 (1974)

[Au2] Aubin, T.: Meilleures constantes dans le théorèm d'inclusion de Sobolev et un théorèm de Fredholm non linéaire pour la transformation conforme de la courbure scalaire. J. Funct. Anal. 32, 148-174 (1979)

[Bec1] Beckner, W.: Sharp Sobolev inequalities on the sphere and the Moser-Trudinger inequality. Preprint, to appear in the Annals of Math.

[Bec2] Beckner, W.: private communication

[Bes] Besse, A.: Einstein Manifolds. Ergebnisse der Mathematik und ihrer Grenzgebiete, 3. Folge, Band 10, Berlin, Heidelberg, New York: Springer 1987

[BØ1] Branson, T., Ørsted, B.: Conformal indices of Riemannian manifolds. Comp. Math. 60, 261-293 (1986)

[BØ2] Branson, T., Ørsted, B.: Conformal deformation and the heat operator. Indiana U. Math. J. 37, 83-110 (1988)

[BØ3] Branson T., Ørsted, B.: Explicit functional determinants in four dimensions. Proc. Am. Math. Soc. 113, 669-682 (1991)

[CY] Chang, S.-Y.A., Yang, P.: Compactness of isospectral conformal metrics on $S^{3}$. Comment. Math. Helv. 64, 363-374 (1989)

[F] Fontana, L.: Sharp borderline estimates on spheres and compact Riemannian manifolds. Ph.D. dissertation, Washington University, 1991

[G] Gilkey, P.: Invariance Theory, the Heat Equation, and the Atiyah-Singer Index Theorem. Wilmington, DE: Publish or Perish 1984

[J] Jensen, G.: Homogeneous Einstein spaces of dimension four. J. Diff. Geom. 3, 309-349 (1969) 
[MS] McKean, H., Singer, I.: Curvature and the eigenvalues of the Laplacian. J. Diff. Geom. 1, 43-69 (1967)

[O'N] O'Neil, R.: Convolution operators and $L(p, q)$ spaces. Duke Math. J. 30, 129-142 (1963)

[On] Onofri, E.: On the positivity of the effective action in a theory of random surfaces. Commun. Math. Phys. 86, 321-326 (1982)

[OV] Onofri, E., Virasoro, M.: On a formulation of Polyakov's string theory with regular classical solutions. Nucl. Phys. B 201, 159-175 (1982)

[OPS1] Osgood, B., Phillips, R., Sarnak, P.: Extremals of determinants of Laplacians. J. Funct. Anal. 80, 148-211 (1988)

[OPS2] Osgood, B., Phillips, R., Sarnak, P.: Compact isospectral sets of surfaces. J. Funct. Anal. 80, 212-234 (1988)

[P1] Polyakov, A.: Quantum geometry of Bosonic strings. Phys. Lett. B 103, 207-210 (1981)

[P2] Polyakov, A.: Quantum geometry of Fermionic strings. Phys. Lett. B 103, 211-213 (1981)

[RS] Ray, D., Singer, I.: R-torsion and the Laplacian on Riemannian manifolds. Adv. Math. 7, 145-210 (1971)

[S] Seeley, R.: Complex powers of an elliptic operator. Proc. Symposia Pure Math. 10, 288-307 (1967)

Communicated by A. Jaffe 